\title{
Simulación del proceso combinado nitrificante y desnitrificante para la remoción de carbono orgánico y nitrógeno
}

\author{
Simulation of combined process for nitrifying and \\ denitrifying removal of organic carbon and nitrogen
}

alngaruca Álvarez, E.; Rojas Zacarías, E.; Vilca Moreno, O.;

Facultad de Ingeniería Química / Universidad Nacional del Centro del Perú

Email: eingaruca@uncp.edu.pe

\section{Resumen}

Los efluentes de la industria pesquera tienen alta concentración de materia orgánica y nitrogenada; su descarga a los cuerpos acuáticos, afecta a la fauna presente en estos ecosistemas. La nitrificación es un proceso biológico que oxida el amonio a nitrato, luego este nitrato puede ser transformado a nitrógeno molecular mediante otro proceso biológico, la desnitrificación. En el presente trabajo, se ha estudiado un sistema combinado desnitrificante-nitrificante cuya configuración ha consistido de un reactor anóxico de filtro y un reactor aerobio de lodo activado. La alimentación de materia orgánica y nitrogenada fue de $1025 \mathrm{mg}$ COT/L y 660 mg N-NH4+ respectivamente, valores promedios obtenidos en el efluente del reactor anaerobio utilizado como primera etapa de tratamiento de las aguas residuales de la industria pesquera.

Se ha formulado un modelo matemático para el sistema combinado, basado en balances de masa y expresiones cinéticas. Las predicciones del modelo se compararon con los resultados experimentales, donde se evaluaron la eficiencia de remoción de materia orgánica y nitrógeno al modificarse la razón de recirculación. El sistema combinado ha operado con diferentes razones de recirculación. $\mathrm{Al}$ aumentar la razón de recirculación hasta un valor de 1.25 , la eficiencia de remoción de materia orgánica y nitrógeno se mantuvieron constante. Para una razón de recirculación de 0.7 , donde se alcanza una velocidad de carga orgánica y nitrogenada de 0.42 y $0.48 \mathrm{~kg} / \mathrm{m} 3 * \mathrm{~d}$, y una razón COT/N-NH4+ de 1 , se logra una eficiencia de remoción de carbono orgánico y nitrógeno de $95 \%$ y $80 \%$, respectivamente. Sin embargo, al aumentar la recirculación hasta 2.35, la eficiencia de remoción de amonio baja drásticamente hasta niveles inferiores a $50 \%$; en cambio, la eficiencia de remoción de carbono orgánico se mantiene sobre $80 \%$. El resultado muestra que con una razón de recirculación hasta 0.7 se logra una remoción simultánea de carbono orgánico y nitrógeno. El modelo predice adecuadamente el comportamiento del sistema.

Palabras clave: simulación, efluente de industria pesquera, nitrificante y desnitrificante, materia orgánica.

\section{Abstract}

Effluents from the fishing industry have a high concentration of organic and nitrogen matter; their discharge to water bodies affects the fauna present in these ecosystems. Nitrification is a biological process that oxidizes ammonia to nitrate, then this can be converted to nitrate nitrogen by other molecular biological process of denitrification. In this work a combined denitrifying-netrifying system has been studied whose configuration consisted of an anoxic reactor filter and an aerobic activated sludge reactor. The supply of organic matter and nitrogen was $1025 \mathrm{mg} \mathrm{CO}$ $\mathrm{T} / \mathrm{L}$ and $660 \mathrm{mg} \mathrm{N}-\mathrm{NH} 4+$, respectively, average values obtained in the anaerobic reactor effluent used as the first stage of treatment of wastewater from the fishing industry.

It has developed a mathematical model for the combined system based on mass balances and kinetic expressions. The model predictions were compared with experimental results where the removal efficiency of organic matter and nitrogen to modify the recirculation ratio were evaluated. The combined system has operated with different reasons for recirculation. By increasing the ratio of recirculation to a value of 1.25 removal efficiency of organic matter and nitrogen they remained constant. For a circulation ratio of 0.7 , where organic and nitrogenous charge speed of 0.42 and $0.48 \mathrm{~kg} / \mathrm{m} 3 * \mathrm{~d}$ is reached, and a COT $/ \mathrm{N}-\mathrm{NH} 4+$ ratio of 1 , a removal efficiency of organic carbon and nitrogen is achieved $95 \%$ and $80 \%$ respectively. However, by increasing circulation to 2.35 , the removal efficiency low ammonium dramatically to levels below $50 \%$, whereas the removal efficiency of organic carbon is maintained over $80 \%$. The result shows that with a ratio of 0.7 to recirculation simultaneous removal of organic carbon and nitrogen is achieved. The model adequately predicts the behavior of the system.

Keywords: simulation, effluents from the fishing industry, nitrifying and desnitrifying, organic matter. 


\section{Introducción}

Existe una gran necesidad de reducir el efecto sobre el medio ambiente que produce la emisión de contaminantes líquidos en una industria. Estos efluentes se tratan para recuperar la materia orgánica particulada y soluble (Roeckel et al., 1994) pero, las aguas residuales resultantes siguen teniendo una alta concentración de materia orgánica, 5-6 g DQO/L (Vidal et al., 1997). Para disminuir esta carga orgánica, se ha propuesto y ensayado un tratamiento anaerobio (Aspe et al., 1997) que reduce una fracción importante de la materia orgánica; sin embargo, se genera una alta concentración de amonio entre $0.2-1.5 \mathrm{~g} \mathrm{~N}-\mathrm{NH} 4+$, siendo necesario un postratamiento para reducir el nivel de amonio y la carga orgánica no eliminada o parcialmente transformada.

El estudio de un modelamiento matemático y su simulación con propósitos de diseño, operación y control del proceso biológico es de suma importancia que permiten predecir el comportamiento del sistema. El modelo matemático es una herramienta para el diseño, operación y control de los procesos. El objetivo de la formulación matemática que se presenta aquí, para el proceso biológico del sistema combinado desnitrificante-nitrificante es para poder contar con una relación matemática que permitan predecir el comportamiento de dicho proceso ante las variaciones de los parámetros que lo rigen. El modelo se hizo con el propósito de que sirvan para el diseño de reactores biológicos con alta concentración de materia orgánica y nitrogenada para cuyo caso se ha considerado el parámetro de diseño carga orgánica volumétrica (Giraldo, E., 1993).

En el caso del diseño del sistema combinado anóxico y aerobio es deseable poder predecir el comportamiento de los sustratos, la eficiencia de remoción de la materia orgánica y nitrogenada en cada uno de los reactores y en el sistema global de tratamiento bajo la geometría de cada uno de los reactores y las condiciones definidas. El modelo que se propone incorpora los modelos cinéticos, la estequiometria de las reacciones, los balances de materia y la descripción de los flujos hidráulicos en cada reactor.

\section{Métodos y materiales}

El modelo es simple por la facilidad de acceder y tiene un número pequeño de variables posibles de modificar. El modelo puede describir el efecto de los parámetros principales de diseño y operación sobre la velocidad de remoción de los sustratos. En el modelo el principal parámetro de diseño que se ha considerado es el tiempo de residencia hidráulica. En una primera parte, una vez establecido el sistema de ecuaciones no lineales se procedió a la calibración del modelo de tal forma que reprodujese las condiciones para un caso base. Las etapas de simulación estarán dadas por:

- Formulación del modelo

- Colección de datos

- Simulación del modelo

- Verificación

- Validación

- Simular Interacciones del Modelo

- Interpretación

- Documentación

\section{Resultados}

Para la primera simulación se realizará con los parámetros determinados de la literatura, las cuales se presentan en la tabla 1 .

\section{Tabla 1}

Valores de parámetros utilizados en el modelo

\begin{tabular}{|c|c|c|}
\hline Parámetro & Valores & Referencias \\
\hline $\mathrm{K}_{\mathrm{N}-\mathrm{NO} 3}^{-}$ & 0.573 & Mardones, 2000 \\
\hline $\mathrm{K}_{\mathrm{N}-\mathrm{NO} 2}^{-}$ & 0.335 & Mardones, 2000 \\
\hline $\mathrm{Ks}_{\mathrm{N}-\mathrm{NO} 3}{ }^{-}$ & 0.54 & Mardones, 2000 \\
\hline $\mathrm{Ks}_{\mathrm{N}-\mathrm{NO} 2}^{-}$ & 0.46 & Mardones, 2000 \\
\hline $\mathrm{Ks}_{\mathrm{CH} 3 \mathrm{COO}}-$ & 18.5 & Grady et al., 1999 \\
\hline $\mathrm{X}_{\text {anox }}$ & 10500 & Puñal, 1998 \\
\hline$Y_{H}$ & 0.6 & Grady et al, 1999 \\
\hline $\mathrm{U}_{\text {max,h }}$ & 7.5 & Furumai, 1992 \\
\hline $\mathrm{Ks}_{\mathrm{CH} 3 \mathrm{COO}^{-}}$ & 10 & Furumai, 1992 \\
\hline $\mathrm{Ko}_{2} \mathrm{~h}$ & 0.1 & Furumai, 1992 \\
\hline$Y_{\text {he }}$ & 0.62 & Makinia, 2000 \\
\hline$Y_{x 01}$ & 0.1875 & Tanaka, 1981 \\
\hline $\mathrm{U}_{\max , \mathrm{ao}}$ & 1.97 & Picioreanu, 1997 \\
\hline $\mathrm{K}_{\text {snh4 }}$ & 1.0 & Furumai, 1992 \\
\hline $\mathrm{K}_{\mathrm{o2,ao}}$ & 0.3 & Picioreanu, 1997 \\
\hline $\mathrm{Y}_{\mathrm{ao}}$ & 0.147 & Picioreanu, 1997 \\
\hline$Y_{\mathrm{xo2}}$ & 0.046 & Picioreanu, 1997 \\
\hline $\mathrm{K}_{\text {inh4 }}$ & 21151.8 & Picioreanu, 1997 \\
\hline $\mathrm{U}_{\max , \mathrm{no}}$ & 1.88 & Picioreanu, 1997 \\
\hline $\mathrm{K}_{\text {sno2 }}$ & 0.511 & Picioreanu, 1997 \\
\hline $\mathrm{K}_{\mathrm{o2,no}}$ & 0.5 & Furumai, 1992 \\
\hline $\mathrm{Y}_{\mathrm{no}}$ & 0.06 & Huang, 1996 \\
\hline$Y_{x 03}$ & 0.017 & Derac, 1983 \\
\hline $\mathrm{K}_{\text {inh4 }}$ & 4152.87 & Picioreanu, 1997 \\
\hline
\end{tabular}

Los resultados de la simulación se compararon con los resultados experimentales del gráfico 4.1 obtenidos en el laboratorio de Bioingeniería de la Facultad de Ingeniería, Departamento de Ingeniería Química de la Universidad de Concepción - Chile (Roeckel et al., 2001). 


\section{Resultados}

\section{Discusión de los resultados de la simulación}

La primera simulación se ha realizado con los parámetros de la Tabla $\mathrm{N}^{\circ} 1$ y el programa fue realizado en el software MatLab.

\section{Figura 1}

Eficiencia de remoción de carbono orgánico en el reactor anóxico y aerobio

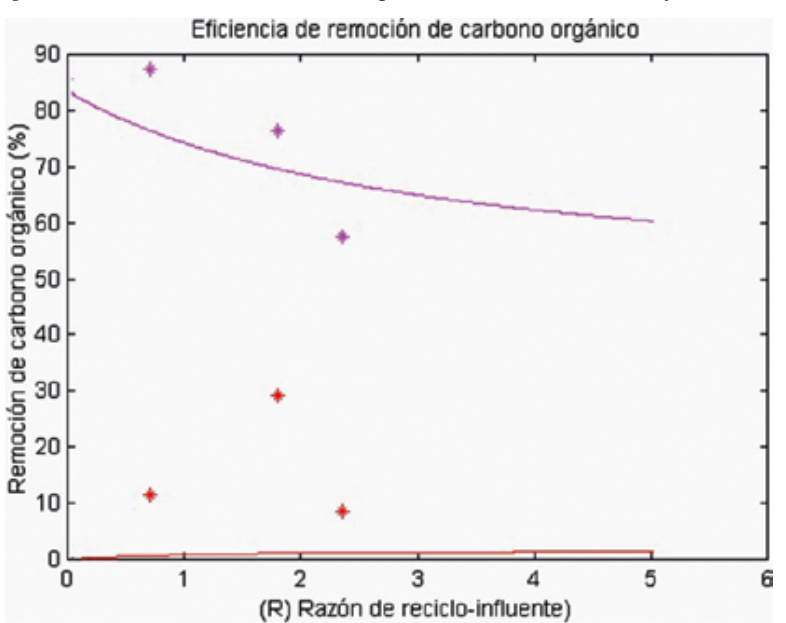

En la segunda etapa se ha simulado el sistema combinado utilizando un programa sin considerar al nitrito como componente intermedio en la desnitrificación y nitrificación, y considerando al reactor nitrificante como un reactor RCPA, además sin considerar el reciclo de lodo desde el decantador. En la tercera etapa de simulación ya se ha considerado las condiciones de operación y de funcionamiento del sistema combinado desnitrificante-nitrificante, en esta parte ya se consideró las cinéticas de inhibición para el proceso de nitrificación, el reciclo de lodo en la desnitrificación, y el comportamiento del reactor nitrificante como un reactor de lodo activado. La figura $\mathrm{N}^{\circ} 2$ se representa los resultados de la simulación comparados con los datos experimentales.

\section{Figura 2}

Eficiencia de remoción de nitrógeno en el reactor anóxico y aerobio

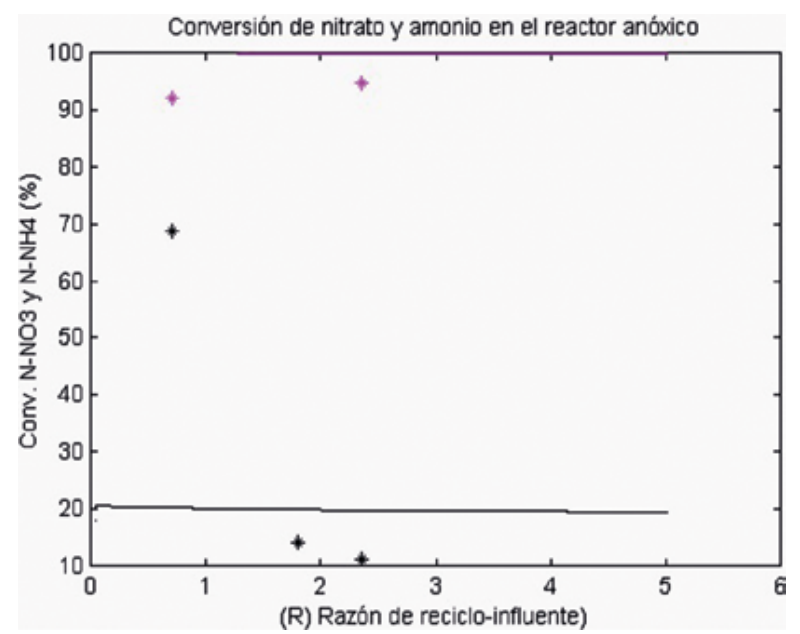

El resultado de la simulación para la eficiencia de remoción de carbono orgánico en el reactor aerobio no concuerda con los datos experimentales cuando se operó con una razón de reciclo de 1,8. En cambio los resultados de la simulación para el caso de la eficiencia de remoción de carbono orgánico en el reactor anóxico convergen adecuadamente a los resultados.

La predicción de la eficiencia de remoción de nitrógeno en el reactor anóxico tiene una buena aproximación para las diferentes razones de recirculación. Sin embargo, para un reciclo de 0.7 la simulación del modelo en predecir la eficiencia de conversón de amonio en el reactor nitrificante se encuentra fuera del dato experimental. Los resultados de la figura 3, corresponden a la razón COT/N-NOx y COT/N-NH4+ tanto experimental y simulado. Según esta figura, el modelo predice muy bien las razones carbono/nitrógeno. Las diluciones de las concentraciones de los componentes por efecto de una mayor recirculación afectan las razones COT/N.

Adicionalmente se hizo un análisis de sensibilidad de la respuesta del modelo a las variables Umax y Ks para cada uno de las reacciones de interés de tal forma que se pudiera tener idea de la importancia relativa de dichas variables sobre las respuestas del modelo. Estos resultados son muy extensos y no se presentan aquí.

La verificación del modelo se hizo utilizando datos experimentales obtenidos en el laboratorio para 4 razones de. Los resultados se pueden ver en la Figura $N^{\circ}$ 1. Como se puede observar los resultados del modelo están muy dispersos comparados con los datos experimentales con excepción de las razones COT/N-NOx y COT/N-NH4+.

\section{Figura 3}

Razón COT/N-NOx y COT/N-NH4+

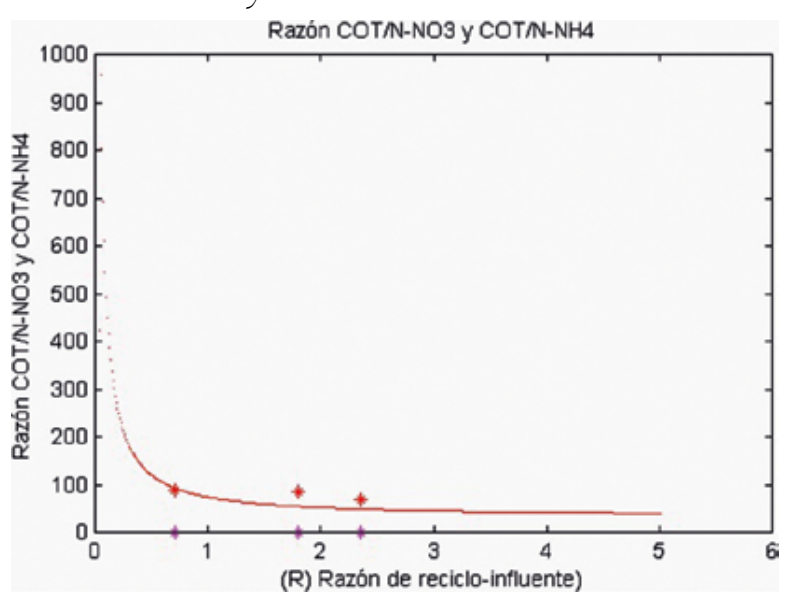

\section{Conclusiones}

- Es importante resaltar el comportamiento de la razón $\mathrm{C} / \mathrm{N}$, donde la curva presenta una declinación cuando se hay una alta eficiencia de remoción de materia orgánica. 
- El comportamiento de la concentración de nitrato y nitrito en el reactor anóxico están muy cercanos a cero.

- El modelo matemático desarrollado es una herramienta poderosa para ganar rápidamente un entendimiento profundo del comportamiento en estado estacionario del sistema combinado.

- El modelo se puede hacerse más complejo incorporando las funciones de inhibición para el caso de la desnitrificación y otros efectos sobre la transferencia de oxígeno en la nitrificación.

\section{Referencias bibliográficas}

Anderson G.K., Kasapgil B., y Ince O., "Microbiological Study of Two-Stage Anaerobic Digestion During Start-Up”, Water Research, 28(11), 2383 2392 (1994).

Banerjee A., Elefsiniotis P. y Tuhtar D., "Effect of HRT and Temperature on the Acidogenesis of Municipal Primary Sludge and Industrial Wastewater", Water Science \& Technology, 38(8-9), 417-423 (1998).

Bernet N., Habouzit F., Moletta R., "Use of an industrial effluent as a carbon source for denitrification of a high-strength wastewater”, Applied Microbiology Biotechnology, 46, 92-97 (1996).

Dee A., James N., Jones I. Strickland J., Upton J., y Cooper P., "Pre- or Post-Denitrification at Biological Filter Works? A Case Study", Water Science \& Technology, 29(10-11), 145-155 (1994).

Guerrero L., Omil F., Méndez R., y Lema J., “Anaerobic Hydrolysis and Acidogenesis of Wastewaters from Food Industries with High Content of Organic Solids and Protein”, Water Research, 33(15), 3281-3290 (1999).

Janssen A., Meijer S., Bontsema J., Lettinga G., "Application of the Redox Potential for Controlling a Sulfide Oxidizing Bioreactor", Biotechnology and Bioengineering, 60(2), 147-155 (1998).
Koster I. W. y Lettinga G., "Anaerobic Digestion at Extreme Ammonia Concentrations", Biological Wastes, 25, 51-59 (1988).

Lay Jiunn-Jyi, Li Yu-You, Noike Tatsuya, "The influence of $\mathrm{pH}$ and ammonia concentration on the methane production in high-solids digestion processes", Water Environmental Research, 70(5), 1075-1082 (1998).

Lovley Derek, Dwyer Daryl, y Klug Michael, "Kinetic Analysis of Competition Between Sulfate Reducers and Methanogens for Hydrogen in Sediments", Applied and Environmental Microbiology, 43(6), 1373-1379 (1982).

Percheron Gilles, Michaud Sébastien, Bernet Nicolas, y Moletta René, "Nitrate and Nitrite Reduction of a Sulfhide-Rich Environment”, J. Chem. Technol. Biotechnol., 72, 213-220 (1998).

Powell Francis E. \& Brooks Brian W., "Reaction Steady States and Stationary States in Modelling Semi-Batch Enzime Hydrolysis", Journal Chemical Technology and Biotechnology, 57, 65-71 (1993).

Rustrian E., Delgenes J. P., Bernet N., y Moletta R. "Nitrate Reduction in Acidogenic Reactor: Influence of Wastewater COD/N-NO3 Ratio on Denitrification and Acidogenic Activity", Environmental Technology, 18, 309-315 (1997)

Rustrian Elena, Delgenes Jean, Bernet Nicolas, y Moletta René, "Simultaneous Removal of Carbon, Nitrogen and Phosphorus from Wastewater by Coupling Two-Step Anaerobic Digestion with a Sequencing Batch Reactor”, J. Chem. Technol. Biotechnol., 73, 421-431 (1998).

Vidal G., Aspé E., Martí M., y Roeckel M., “Treatment of recycled wastewaters from fishmeal factory by an anaerobic filter”, Biotechnology Letters, 19(2), 117-121 (1997).

Yu Hanqing, Wilson Francis, y Tay Joo-Hwa, "Kinetic Analysis of an Anaerobic Filter Treating Soybean Wastewater", Water Research, 32(11), 3341-3352 (1998) 